\title{
Correction to: Global terrain classification using 280 m DEMs: segmentation, clustering, and reclassification
}

\author{
Junko Iwahashi ${ }^{1 *}$, Izumi Kamiya ${ }^{2}$, Masashi Matsuoka ${ }^{3}$ and Dai Yamazaki ${ }^{4}$
}

\section{Correction}

In the publication of this article (Iwahashi et al. 2018), there was an error in Table 3 in terrain group 10, 11 and 12 .

The error: "natural level".

Should instead read: "natural levee".

This has now been updated in the original article (Iwahashi et al. 2018).

\section{Author details}

'Geospatial Information Authority of Japan, Geography and Crustal Dynamics Research Center, Kitasato 1, Tsukuba, Ibaraki 305-0811, Japan. ${ }^{2}$ Japan Digital Road Map Association, Hirakawa 1-3-13, Chiyoda-ku, Tokyo 102-0093, Japan ${ }^{3}$ Interdisciplinary Graduate School of Science and Technology, Tokyo Institute of Technology, Nagatsuta 4259-G3-2, Midori-ku Yokohama, Kanagawa 226-8502, Japan. ${ }^{4}$ Institute of Industrial Science, The University of Tokyo, Tokyo, Japan.

Published online: 23 February 2018

\section{Reference}

Iwahashi J, Kamiya I, Matsuoka M et al (2018) Global terrain classification using

280 m DEMs: segmentation, clustering, and reclassification. Prog Earth Planet Sci 5:1 https://doi.org/10.1186/s40645-017-0157-2

\footnotetext{
* Correspondence: iwahashi-j96pz@mlit.go.jp

${ }^{1}$ Geospatial Information Authority of Japan, Geography and Crustal Dynamics Research Center, Kitasato 1, Tsukuba, Ibaraki 305-0811, Japan
} 
Table $3 C R^{a}$ and $S C^{b}$ of geomorphological and geological units for each terrain group in Japan

\begin{tabular}{ll}
\hline Terrain group & Landform element of JEGM \\
\hline $1 \mathrm{a}$ & Mountain (84\%), volcano ${ }^{c}(8 \%)$, others (8\%)
\end{tabular}
Geological unit of SDGM

Accretionary complex (30\%), mafic volcanic rocks (Jurassic to Pleistocene) (20\%), plutonic rocks (Silurian to Middle Miocene) $(13 \%)$, felsic volcanic rocks $(9 \%)$, metamorphic rocks $(9 \%)(\times 2.3)$, others (19\%)

$1 b \quad$ Mountain (53\%), volcano $(32 \%)(\times 7.0)$ volcanic footslope $(9 \%)$, others (6\%) valley bottom lowland $(10 \%)(\times 2.0)$, volcanic footslope $(5 \%)$, others $(15 \%)$ volcanic footslope (9\%), valley bottom lowland (8\%), terrace covered with volcanic ash soil $(6 \%)$, volcanic hill $(6 \%)(\times 2.4)$, others $(9 \%)$ Volcano $(55 \%)(\times 12.5)$, volcanic footslope $(36 \%)(\times 7.7)$, others $(9 \%)$

Volcanic footslope $(60 \%)(\times 12.8)$, gravelly terrace $(10 \%)$, terrace covered with volcanic ash soil $(9 \%)(\times 2.3)$, volcano $(6 \%)$, others (15\%)

Terrace covered with volcanic ash soil $(19 \%)(\times 4.8)$ gravelly terrace $(16 \%)(\times 2.4)$, valley bottom lowland $(14 \%)(\times 3.0)$, hill $(12 \%)$, volcanic footslope $(7 \%)$, back marsh $(7 \%)(\times 2.5)$, alluvial fan $(7 \%)(\times 2.3)$, delta and coastal lowland $(5 \%)(\times 3.1)$, others $(13 \%)$ volcanic ash soil $(14 \%)(\times 3.5)$, hill $(13 \%)$, alluvial fan $(12 \%)(\times 4.3)$, valley bottom lowland $(10 \%)(\times 2.2)$, volcanic footslope $(8 \%)$, others $(14 \%)$

Terrace covered with volcanic ash soil $(19 \%)(\times 4.8)$ alluvial fan $(16 \%)(\times 5.7)$, gravelly terrace $(15 \%)(\times 2.2)$, back marsh $(14 \%)(\times 5.2)$,

delta and coastal lowland $(8 \%)(\times 4.7)$, others $(28 \%)$

Alluvial fan (30\%) $(\times 10.5)$, gravelly terrace $(30 \%)(\times 4.4)$, terrace covered with volcanic ash soil $(16 \%)(\times 4.1)$, volcanic footslope $(10 \%)(\times 2.1)$, others $(14 \%)$

Back marsh (28\%) (×9.9), alluvial fan $(22 \%)(\times 7.5)$, delta and coastal lowland $(14 \%)(\times 8.3)$, gravelly terrace $(8 \%)$, natural levee $(7 \%)(\times 11.2)$, others $(21 \%)$

Back marsh $(36 \%)(\times 13.1)$, delta and coastal lowland $(18 \%)(\times 10.9)$, alluvial fan $(12 \%)(\times 4.3)$, natural levee $(11 \%)(\times 17.1)$, reclaimed land $(6 \%)(\times 13.3)$, others $(17 \%)$

Back marsh $(56 \%)(\times 20.0)$, delta and coastal lowland $(21 \%)(\times 13.0)$, natural levee $(14 \%)(\times 21.0)$, reclaimed land $(7 \%)(\times 14.7)$, others $(2 \%)$
Mafic volcanic rocks (Jurassic to Pleistocene) $(39 \%)(\times 3.1)$ accretionary complex (13\%), Plutonic rocks (Silurian to Middle Miocene) (10\%), sediments (8\%), felsic volcanic rocks (7\%), pyroclastic flow deposits (6\%)

Accretionary complex (25\%), mafic volcanic rocks (Jurassic to Pleistocene) (18\%), plutonic rocks (Silurian to Middle Miocene) (13\%), sediments (13\%), felsic volcanic rocks (12\%), sedimentary rocks (Silurian to Middle Miocene) (7\%), metamorphic rocks (6\%), others $(6 \%)$

Sediments (23\%), accretionary complex (22\%), mafic volcanic rocks (Jurassic to Pleistocene) (17\%), sedimentary rocks (Silurian to Middle Miocene) $(13 \%)(\times 2.3)$, felsic volcanic rocks $(9 \%)$, plutonic rocks $(6 \%)$, others $(10 \%)$

Sediments (38\%), accretionary complex (13\%), Plutonic rocks (Silurian to Middle Miocene) (12\%), mafic volcanic rocks (Jurassic to Pleistocene) (9\%), pyroclastic flow deposits (8\%), sedimentary rocks (Silurian to Middle Miocene) (7\%), felsic volcanic rocks (7\%), others (6\%)

Sediments (54\%), pyroclastic flow deposits $(13 \%)(\times 2.3)$, accretionary complex (7\%), sedimentary rocks (Silurian to Middle Miocene) (7\%), mafic volcanic rocks (Jurassic to Pleistocene) $(5 \%)$ others (14\%)

Mafic volcanic rocks (Holocene) $(47 \%)(\times 84.3)$, volcanic debris (Miocene to Holocene) $(21 \%)(\times 12.6)$, mafic volcanic rocks (Jurassic to Pleistocene) (16\%), pyroclastic flow deposits (8\%), others (8\%)

Sediments (35\%), volcanic debris (Miocene to Holocene) $(23 \%)(\times 13.9)$, pyroclastic flow deposits $(18 \%)(\times 3.1)$ mafic volcanic rocks (Holocene) $(14 \%)(\times 25.8)$, mafic volcanic rocks (Jurassic to Pleistocene) (7\%), others (3\%)

Sediments $(79 \%)(\times 2.3)$, pyroclastic flow deposits $(8 \%)$ others (13\%)

Sediments $(81 \%)(\times 2.4)$, pyroclastic flow deposits $(5 \%)$ others (14\%)

Sediments $(95 \%)(\times 2.8)$, others $(5 \%)$

Sediments $(86 \%)(\times 2.5)$, pyroclastic flow deposits $(5 \%)$, others (9\%)

Sediments $(99 \%)(\times 2.9)$, others $(1 \%)$

Sediments $(100 \%)(\times 2.9)$

Sediments $(100 \%)(\times 2.9)$

Legends with $C R$ of $5 \%$ or more are shown. Clusters with SC over $\times 2.0$ are shown in bold text and the factors are in parentheses

${ }^{a}$ Composition ratio

${ }^{b}$ specialization coefficient

climited to Holocene volcano in JEGM 